\title{
REVIEWER ACKNOWLEDGMENTS
}

The Editor and Associate Editors wish to thank the following persons who served as principal reviewers for the Journal of Counseling Psychology by reviewing a minimum of four manuscripts.

\section{PRINCIPAL REVIEWERS}

Juan Martin Gómez Penedo

Cindy Lee Juntunen

Katie Aafjes-van Doorn

Roberto L. Abreu

Hector Y. Adames

Blake A. Allan

Jeffrey S. Ashby

John Auerbach

Germine H. Awad

Ruth A. Baer

Kim Baranowski

Avigail Bar-Sella

Tal Ben-David Sela

Sarah Bloch-Elkouby

Jan Boehnke

Rachel E. Brenner

James Brooks

Daniel Brossart

Joshua Eusty Jonathan Buckman

Stephanie L. Budge

Germán A. Cadenas

Christian D. Chan

Roei Chen

Kirsty Clark

Kevin Cokley

Lynn H. Collins

Michael J. Constantino

Corey Cook

Giulio Costantini

Alice E. Coyne

Ryan Cragun

Melissa Miléna De Smet

Cirleen DeBlaere

Eric D. Deemer

Frank R. Dillon

David M. Dunkley

Robert Elliott

Devin English

Fredrik Falkenström

Nick Firth

Jillian Fish

Shimrit Fisher

Megan Foley-Nicpon

Anjali Forber-Pratt

\author{
Yogev Kivity
}

Linh P. Luu

\section{CONTRIBUTING REVIEWERS}

Katherine Jean Forney

Ze'ev Frankel

Myrna L. Friedlander

Ramaris German

Monica A. Ghabrial

Abbie E. Goldberg

Jeffrey A. Hayes

Laurie Heatherington

Richard Henry

Clara E. Hill

Peter Hilpert

Aaron Hogue

Kimberly Hook

Sharon G. Horne

William T. Hoyt

Carla D. Hunter

Evelyn A. Hunter

Ayse Ikizler

Zac E. Imel

Derek Kenji Iwamoto

Margo A. Jackson

Skyler D. Jackson

Morgan C. Jerald

LaRae M. Jome

Shanna Kattari

Michael Katz

Jack Keefe

Gulsah Kemer

L. B. Klein

Virpi-Liisa Kykyri

Jae Lee

Runze Lee

Fangsong Liu

Ido Liviatan

Katie Loth

Patrick Luyten

Danielle Magaldi

Bailee Malivoire

Tyler Mason

Emmie Matsuno

Andrew McAleavey
Julian Rubel

Sherri L. Turner

\author{
Shannon McClain \\ Ryon C. McDermott \\ Dan McNeish \\ Ethan H. Mereish \\ Erhard Mergenthaler \\ Melissa L. Morgan Consoli \\ Della V. Mosley \\ Craig Nagoshi \\ Christoph Niepel \\ Helene A. Nissen-Lie \\ Kari O'Grady \\ Yuki Okubo \\ Tiffany A. O'Shaughnessy \\ Jesse Owen
}

Brian T. Pace

Eleanor Pardess

Paul B. Perrin

Alex L. Pieterse

V. Paul Poteat

Mary Pritchard

Amy L. Reynolds

Michele Rivas

Michael Robbins

David Rosmarin

Rob Saunders

David Saxon

Jillian R. Scheer

Shirley Serfaty

Jaclyn Siegel

Jesse M. Smith

Laura Smith

Nili Solomonov

Eric Swank

Giorgio A. Tasca

Terence J. G. Tracey

Rivka Tuval-Mashiach

Tracy L. Tylka

Dana Tzur-Bitan

Cindy Veldhuis

Elizabeth M. Vera

Melanie M. Wilcox 
CONTRIBUTING PREDOCTORAL CO-REVIEWERS

Hannah Appleseth

Priya Bansal

Sergio Dominguez

Allison Drake

Maddie Egli

Alvaro Gamio Cuervo
Kamryn Holtz

Melanie Miller

Destin Mizelle

Savannah Pham

Nicole Porter

Garrett Ross
Melinda Troyka

Shelby D. Tuthill

Nathaniel Woznicki

Jody Zhong 